\section{Polycarbonate Capillaries Replace Glass Tubes to Enhance Safety and Flexibility of Fiber Optics}

Unless you are a committed traditionalist, you are probably reading a lot of material online these days. For that, you can thank telecom fiber optics. But many important medical and industrial applications rely on fiber optics as well: high-power uses such as endoscopic surgery and semiconductor manufacturing call for hollow-core fibers, which avoid damage by carrying most of the light in their hollow centers. Hollow-core fibers have proven highly useful, but they have one serious drawback: they require an external supporting capillary tube, which is usually made of glass. If this tube breaks, the fragments can cause serious or even fatal damage to the biological or solid-state environment. In an article in the May 15 issue of Optics Letters (p. 1373), a group of researchers led by Y.-W. Shi of Fudan University in China reported the fabrication of hollow fibers with capillaries made from polycarbonate (PC), which is more flexible than glass and does not release fragments when it breaks.

Shi, along with colleagues M. Nakazawa and Y. Matsuura of Tohoku University in Japan and K. Iwai and M. Miyagi of Sendai National College of Technology in Japan, began with commercially available PC tubes with 11-mm inner diameters as preforms, and then drew them at a temperature of $208^{\circ} \mathrm{C}$ to an inner diameter of $700 \mu \mathrm{m}$ using glass-drawing techniques. They next coated the inner surface of the tubes with silver and cyclic olefin polymer (COP) using liquid-phase coating methods, in order to reduce the fiber loss at specific optical wavelengths. The resulting PC-capillary-supported hollow fibers showed almost identical transmission characteristics to glass-capillary-supported fibers, with only marginally higher bend and attenuation losses for light with a wavelength of $2-5 \mu \mathrm{m}$.

Although the fiber is designed to deliver these infrared wavelengths, medical applications usually need fibers to also guide optical pilot beams that assist surgeons in aiming the infrared radiation. Shi's group found that the silver/COP coating in combination with the PC tube displayed relatively low loss for red and green pilot beams, although this result was sensitive to the fiber-drawing temperature. These results suggest that hollow fibers with flexible, non-fracturing polycarbonate capillaries may soon replace standard glass-supported fibers and make endoscopy and certain manufacturing techniques safer and easier.

COLIN MCCORMICK

\section{Light-Driven Logic Gates Fabricated from Dye-Sensitized Solar Cell}

Because of their low cost of production, dye-sensitized solar cells offer a promising alternative to solid-state photovoltaic devices. These cells consist of a thin layer of nanocrystalline titania doped with a ruthenium-based dye supported on a conducting electrode and fully immersed in an electrolyte of iodide solution. A counter electrode seals the whole system. Photocurrent is produced when the dye molecules inject electrons into the titania layer after absorbing visible light, and the electrolyte at the other end of the circuit is reduced at the counter electrode and oxidized back after transferring an electron to the dye. In the May 5 issue of Angewandte Chemie International Edition (p. 3143; DOI: 10.1002/anie.200600076), L. Furtado and co-workers at Universidade de Sao Paulo in Brazil demonstrated that by using a dye that accepts rather than injects electrons into the titania layer, the direction of the photocurrent can be controlled by the wavelength of the incident light, allowing fabrication of light-driven logic gates. As ruthenium acetate trinuclear clusters have favorable reduction potential for such a mechanism, the researchers used a complex based on that as a dye, $\left[\mathrm{Ru}_{3} \mathrm{O}(\mathrm{Ac})_{6}(\mathrm{py})_{2}\left(\mathrm{pzCO}_{2} \mathrm{H}\right)\right] \mathrm{PF}_{6}$.

\section{Understand your surface...}

with confocal laser scanning microscopy.

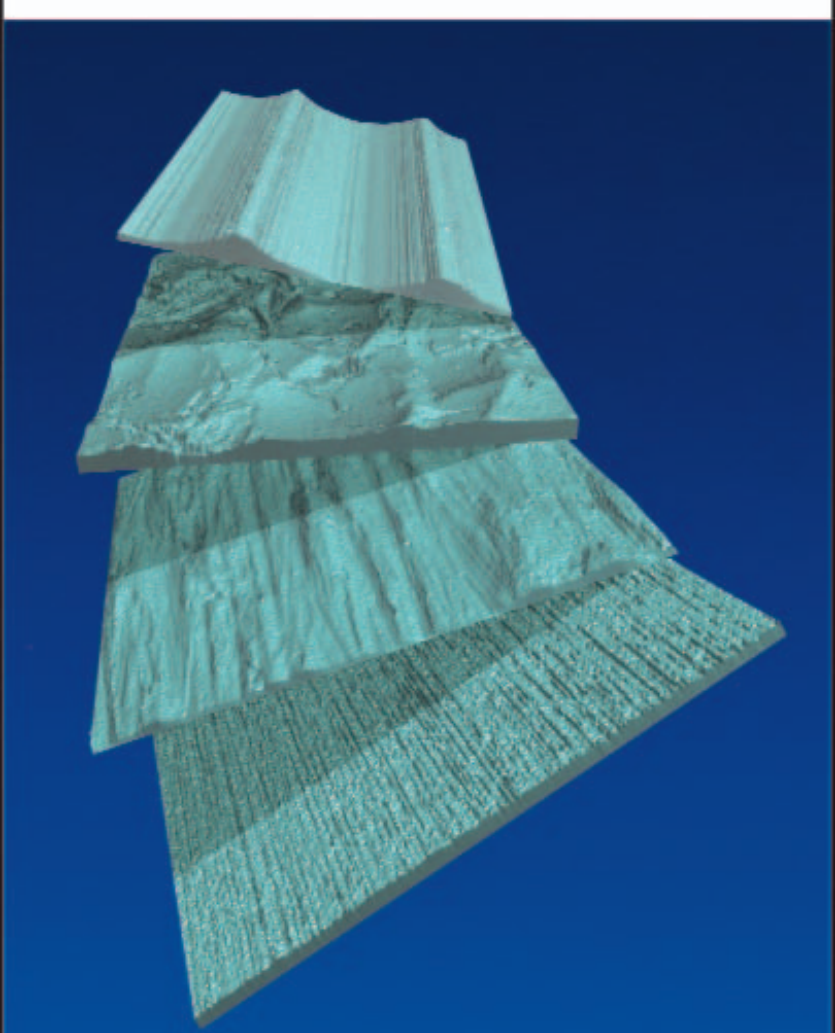

Other techniques only scratch the surface. Get the whole picture using Zeiss LSM 5 PASCAL.

-2D Roughness measurement

- 3D Roughness measurement

- Surface topography

- Volumetric measurement

Get the most from your tools.

- Non-contact

- Multimode confocal imaging

- High resolution

- Large scan areas

- Image archiving

- Extended focus

- Multiple contrast techniques

- High quality optical microscopy

Carl Zeiss

Microlmaging, Inc.

Thornwood, NY

800.233 .2343

micro@zeiss.com

zeiss.com/materials

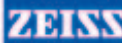

We make it visible. 